\title{
One Micro-thrust Measurement Method Based on the Acoustoelastic Theory
}

\author{
Yang $\mathrm{Ou}^{\mathrm{a}}$, Jianjun Wu, Yu Zhang, Jian Li and Sheng Tan \\ College of Aerospace Science and Engineering, National University of Defense Technology, 410073, Changsha, \\ P.R.China
}

\begin{abstract}
Concerning the traditional way of micro-thrust measurement cannot get the transient thrust value with high precision and the measuring process of piezoelectric sensors is sensitive to the electromagnetic interference, this paper builds one new measurement method basing on the acoustic elasticity theory. The acoustoelastic element is the core of the whole system as it can transform the thrust changes into the variation of acoustic time. Thus adopting a timer to record such variation so that the thrust changes in real-time could be obtained, which is helpful for studying the mechanism of micro thrusters. This method built in this paper is not only noncontact and non-destructive but also easy to operate with simple structure and high precision.
\end{abstract}

\section{Introduction}

As we known, large satellites often have long development cycle with high cost and complex technology, which greatly limits their application and development. Meanwhile, by providing a train of small, discrete impulse bits, micro-thrusters are ideal for such diverse applications as orbit raising, drag makeup, attitude control, constellation station keeping and deep-space missions. Micro-thrusters are especially well-suited for low-thrust, on-board propulsion because they use a non-toxic propellant and are highly reliable, solid-state, low power, and lightweight, as they require no propellant storage system [1].

Therefore, in recent years, many agencies, including the European Space Agency, Iranian Space Agency and Australian National University, have developed several forms of microsatellite systems with its advantages of micro size, light weight, short response time, high function density ratio and cost-effective. With the rapid expansion of micro satellite, micro satellite orbit maneuver, precise attitude control, atmospheric drag compensation and high precision formation flying ability all need such one thruster with small power, light weight and simple structure. The plasma thruster is not only the ideal propulsion scheme but also the key subsystem for attitude controlling, position keeping and orbit maneuver of micro satellite.

Through high precision and resolution measuring the micro thrust and micro impulse, it can get more discrete but precise enough pulse with the control of thrust. And then, such could guarantee the high precision attitude control, which contributes to the relative technologies. However the thrust produced by micro-thrusters is generally pretty small, which ranges from $\mathrm{nN}$ level to $\mathrm{mN}$ level. For example, the thrust produced by pulsed plasma thrusters is generally bovine micro scale. And ESA's

acorresponding author : naihe620@163.com 
LISA project uses a milli bovine scale thruster. In addition, the NASA ST7 mission uses a 2 20 N thruster with a precision of less than $0.1 \mathrm{~N}$ [2] [3].

Although it is relatively easy to measure the thrust in the range of $\mathrm{mN}$ magnitude, however, with thrust decreases, accurate measurements of the thrust of the $\mathrm{nN}$ and $\mu \mathrm{N}$ level become very difficult. On the one hand, the noise of the instrument could affect the accuracy; on the other hand, it will reduce the precision if transient force that passes to the platform remains pending. General speaking, it is urgent to further improve the accuracy of the thrust measurement, especial the thrust measurement in range of the $\mu \mathrm{N}$ level, as for the development of micro satellites [4].

The thrust measurement consists of average thrust and impulse measurement. The essence is to observe the mechanical behaviour of the thrust the thrust stand under the micro-thrust for measuring the thrust indirectly. Furthermore, according to different thrust range, measurement methods are different. For $\mathrm{mN}$ to $\mathrm{N}$ level, the weighing experiment rig and the tilting structure are adopted [5]. While for $\mu \mathrm{N}$ level, it is necessary to take the twist structure, double pendulum structure and the four arm counterweight structure into consideration.

At present, the micro thrust / micro impulse measurement methods with high precision are relatively scarce. In the past, impulse and instantaneous thrust measurement systems typically used an inductive proximity probe, and accelerometer, a linear voltage differential transformer, a differential plate capacitance system, or an optical angular readout. The most sensitive of these past devices appears to be the optical angular readout from Ref. 3 designed in 1968 at NASA Jet Propulsion Laboratory, which can measure thrust as low as $5 \mu \mathrm{N}$ with loads up to $12 \mathrm{~kg}$. A comparable thrust stand was designed at Goddard Space Flight Centre in 1970 that measured thrust as low as $25 \mu \mathrm{N}$, thrust stand deflection as low as $25 \mathrm{~nm}$, and measured the thrust vector. Larger thrust and impulse bit measurement have been made as well. A $5 \mathrm{~N}$ thrust stand was developed by Hagg at NASA Lewis Research Centre in 1989 and impulse bits near 1 Ns were measured at Princeton University [2].

However, there is no high accuracy measurement system at present. It's urgent to raise the measurement accuracy of the measurement as exact as possible for promoting the development of micro-satellites. This paper would build a new thrust measurement system of micro-thrusters with high precision, which basing on the acoustic elasticity principle. This method can get the internal stress of the acoustic elastic element by measuring the variation of sound velocity. And then, the specific values of the thrust are obtained. The key components of this system include a lever table, an ultrasonic transducer, a data acquisition device, and a necessary signal processing system. This system, basing on the relationship between stress and acoustic speed, adopts one visualization method with the special elastic material which could achieve the aim of recording the variation of the micro-thrust.

\section{Acoustoelastic theory}

The acoustoelastic pulse-echo method that has gained wide application in industry was first described by Benson and Raelson in their fundamental 1959 study. The authors proposed a new method for measuring the stresses in an isotropic material. The phenomenon that the method is based on is in the difference of the velocities of transverse waves polarized parallel and perpendicular to the direction of stresses in the materials under load, i.e., the anisotropy of the stressed medium. This phenomenon has been termed the acoustoelastic effect and method for assessing the stress-strain state of structural elements is referred to as the acoustoelastic method [6].

And then, a number of studies have been done around the acoustoelastic method. Cantrell and Salama focused on inhomogeneous stress determination and on material characterisation for recent developments in non-linear acoustelasticity that implicates directly the thermoelastic properties and in appropriate cases the magneto elastic properties of multiphase materials. Dubuget proposed an original ultrasonic immersion device which permits the determination of the elastic constants by velocity measurements for tow aluminium alloys and the third-order elastic constants have been obtained from acoustoelastic responses [7]. However, seldom is such method applied to measuring the thrust, more application are in stress testing. 
Acoustic elasticity is a branch of mechanics that studies the interaction of structures, fluids, and sound fields. It involves ships, underwater weapons, aircraft, automobiles, buildings, chemicals, marine and water conservancy projects, and many other fields. It will radiate sound waves to surrounding or inner media when elastic structures are forced to vibrate under the external thrust. Meanwhile, it could make the vibration or the thrust change as the acoustic wave loads in the elastic structure in the form of feedback. Therefore, it's the interaction of these that builds a coupled system between the excitation, structure and acoustics. And the main task of the acoustic elasticity theory is to detect and understand the relationship among the basic mechanical and acoustic, in addition to analyse the characteristics and regularities of the structure induced vibration and sound radiation. Such method is not only a non-destructive method for measuring stress, but also one new means to analysis the nontransparent material's stress as for its reliability, toughness, economical efficiency and easy-using. The wave velocity changes in accordance with the stress; therefore, we can get specific value of the stress by measuring the variation of wave velocity. What's more, if the same distance is set, we can get different sonic time in order to reflect the variation of the stress.

However, seldom is such method applied to measuring the thrust, more application are in stress testing. And the goal of this study is to obtaining the thrust data on the spatial and temporal dynamics of the variations when the micro-thrusters work.

As we can know from the former research, the transversal wave velocity, polarised along the direction of the stress, differs from that for which the polarisation is perpendicular to the stress. Therefore, by using measurement of the wave velocity in a material, in the absence or in the presence of stresses and with the knowledge of the different elastic constants, the applied stress on the solid can be determined. We note that the third-order elastic constants, the applied stress on the solid can be determined. We note that the third-order elastic constants are neglected in case of the linear elasticity. Moreover the exploitation of the acoustoelastic phenomenon is quite difficult since the influence of stresses on the ultrasonic velocity is very weak and also since the crystallite orientation or the texture and the microstructure have a strong influence on the ultrasound propagation.

The application of a stress on an isotropic and homogeneous body leads to a modification of its mechanical behaviour. In the presence of such a phenomenon, it has been shown that the propagation velocity of ultrasound waves propagating in the $\mathrm{x}$-direction. And polarized along different directions are expressed by the following acoustoelastic equations [8] [9] [10].

Longitudinal wave propagating along the $\mathrm{x}$-direction:

$$
\rho_{0} V_{x x(x)}^{2}=\lambda+2 \mu+\frac{\sigma}{3 \lambda+2 \mu}\left[\frac{\lambda+\mu}{\mu}(4 \lambda+10 \mu+4 m)+\lambda+2 l\right]
$$

Transversal wave propagating along $\mathrm{x}$ and polarised along the $\mathrm{y}$ - or $\mathrm{z}$-direction:

$$
\rho_{0} V_{x y(x)}^{2}=\rho_{0} V_{x z(x)}^{2}=\mu+\frac{\sigma}{3 \lambda+2 \mu}\left[4 \lambda+4 \mu+m+\frac{n \lambda}{4 \mu}\right]
$$

In the above equations, $\rho_{0}$ is the density in the initial state. $V_{x x}, V_{x y}$ and $V_{x z}$ are, respectively, the propagation velocity of the ultrasound waves along the $\mathrm{x}$-direction with a particle displacement along the $\mathrm{x}$-, $\mathrm{y}$ - and z-directions. $\lambda$ and $\mu$ are the elastic constants or Lame constants. $l, \mathrm{~m}$ and $\mathrm{n}$ are the third-order elastic constants or Murnaghan constants. $\sigma$ represents the axial stress [11] [12] [13].

From the equations, we know the propagation velocity of sound waves is inconsistent with the change of stress in different directions and the longitudinal wave shows the strongest sensitivity to the stress change along the direction of wave propagation. Therefore, the position of acoustic elastic element and acoustic emitter is set up to ensure that the longitudinal wave is parallel to the stress direction and satisfies the relationship (1):

$$
\rho_{0} V^{2}=\lambda+2 \mu+\frac{\sigma}{3 \lambda+2 \mu}\left[\frac{\lambda+\mu}{\mu}(4 \lambda+10 \mu+4 m)+\lambda+2 l\right]
$$

The velocity is generally obtained by measuring the propagation time of equidistant distances:

$$
V=\frac{L}{t}
$$


Where $\mathrm{L}$ is the distance and $\mathrm{t}$ is the propagation time.

From the mechanics of materials, we also get the relationship between the internal force and stress:

$$
\sigma=\frac{F}{S}
$$

Where $\mathrm{F}$ is the internal force and $\mathrm{S}$ is the cross-section.

Simultaneous these formulas, we can get the relationship between the force and the propagation time:

$$
F=\frac{\mu S\left(\rho_{0} L^{2}-\lambda t^{2}-2 \mu t^{2}\right)(3 \lambda+2 \mu)}{t^{2}\left(4 \lambda^{2}+15 \lambda \mu+4 m \lambda+4 m \mu+2 l \mu+10 \mu^{2}\right)}
$$

Control the loading so that the thrust is equal to the internal force so that we can get the thrust by measuring the propagation.

\section{Basic structure}

In this paper, the acoustic elastic element is regarded as the core of the system as it can reflect the variation of thrust through the change of time. And such measurement method is built according to the characteristics of micro thruster and acoustic elastic theory. On the basis of the system, we can obtain the real-time change of the thrust when using high-speed signal collector to record the value between the signals. This measurement is non-contact and non-destructive for a sensor or other measuring devices installed on the thrusters are not needful. Therefore, the system reduces the interference of mechanical vibration and power supply cables while solves problem like zero drift and unstable equilibrium position calibration, difficulty and low accuracy. General speaking, it has the advantages of simple structure, easy operation and high reliability while the most important thing is to provide a method to solve the problem of the traditional thrust measuring method which is difficult to measure the transient thrust[14] [15] [16].

The instantaneous thrust measurement system basing on the principle of acoustic elasticity consists of four parts, which are acoustic environment system, thruster system, control system, signal data collector and signal processing system. The overall composition is shown in Figure 1.

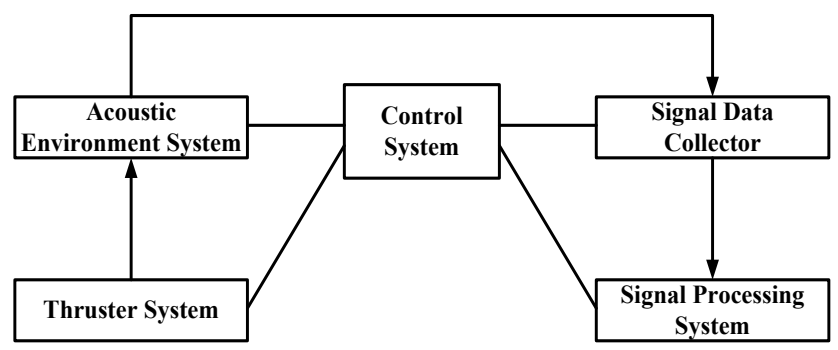

Figure 1. Overall composition.

\subsection{Acoustic environment system}

As shown in Figure 2, the acoustic environment system includes an organic glass wedge 1, a transmitting probe 2 , a sound absorption material plate 3 , an acoustic elastic element 4 , a fixing device 5 , and a receiving probe 7 . In addition, the acoustic elastic element 4 can be replaced according to the different thrust so as to meet the measurement requirements. The transmitting probe 2 is used to produce sound waves while the receiving probe 7 mainly completes the filtering and wave acquisition. The Plexiglas wedge is a low velocity medium, and the acoustic elastic element is a high velocity medium. A sound absorbing material is used to absorb longitudinal waves reflected in a wedge by multiple reflections at the interface. The fixing device ensures the stability of each device [17] [18]. 
The transmitting probe transmits ultrasonic, and then it will produce LCR wave and refracted shear wave when the ultrasonic is incident on an interface between low wave velocity medium and high velocity wave medium at the first critical angle. The two waves have the same frequency and would be received by the receiving probe after multiple reflections. The LCR wave will precede the other ultrasonic clutter to the receiving probe due to its unique characteristics. The sound absorbing material board selects the sound waves which are not qualified so as to ensure that the sound waves needed for the measurement are left. The fixing device maintains the acoustic environment system stable and does not affect the normal operation of the acoustic component by movement.

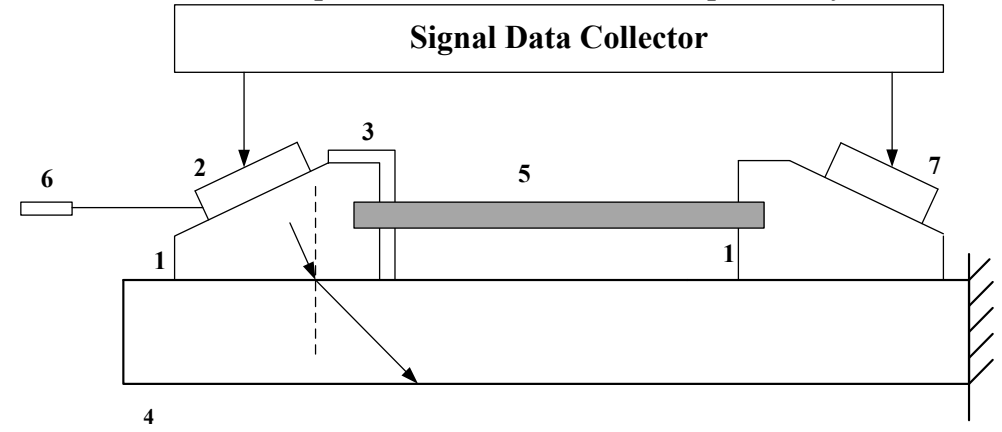

1-Organic Glass Wedge; 2-Transmitting Probe; 3-Sound Absorption Material Plate;

4-Acoustic Elastic Element; 5-Fixing Device; 6-Timing Trigger; 7-Receiving Probe

Figure 2. Acoustic environment system.

The main function of the system is to create an acoustic environment for the acoustic elastic element so that the stress change of the acoustic elastic element can be reflected by the wave velocity.

\subsection{Thruster system}

The thruster system is shown in Figure 3, which includes a micro-thruster 8 and a lever 9. The purpose of this paper is to realize the force measurement. Therefore, different types of thrusters can be replaced according to the requirements of the force measurement without considering the structure and function of the thruster. Pulsed plasma thruster is taken as an example. The lever 9 and the acoustic elastic element 4 are semi-rigid connected, and the lever transmits and amplifies the thrust.

The sound velocity variation of elastic element is directly related to the thrust's change. The lever can amplify the thrust in an appropriate ratio, and then provides a larger thrust for recording the velocity variation more precisely. Finally, real data is obtained by scaling the value according to the lever parameters.

The main function of this system is to generate thrust and to amplify and transfer the thrust to the acoustic elastic element so that it is in a stress state.

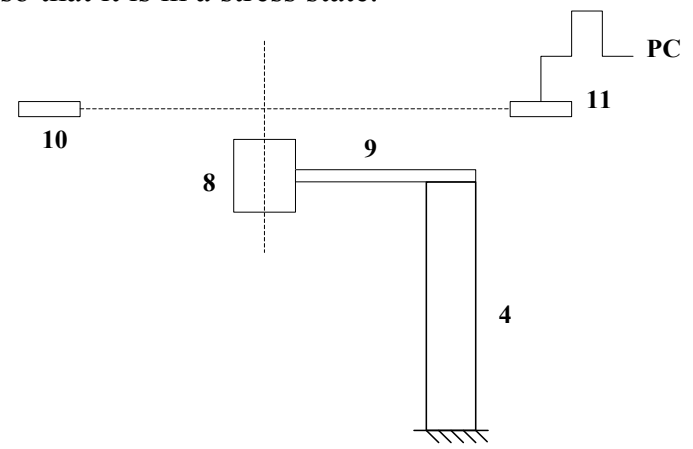

4-Acoustic Elastic Element; 8-Micro-thruster; 9-Level; 10-Electromagnetic

Emitter; 11-ELectromagnetic Trigger

Figure 3. Micro-thruster system. 


\subsection{Control system}

The ultrasonic wave emitted by the emission probe in Figure 2 does not exist continuously; otherwise the probe signal is chaotic. So it is necessary to make the transmitting probe timing trigger in a probe recognized frequency. The specific thrust calculated by this method is assumed to be approximately equivalent to the thrust loaded from the micro-thruster at the same moment as the deformation of elastic element is little. Therefore, a timing trigger is used here to realize the timing of transmitting ultrasonic waves.

Most of the thrusters are not continuous but pulsed. So it would be uneconomical if ultrasonic waves are still emitted when the micro-thruster doesn't work. This paper mainly aims at the plasma thruster which gets the thrust by spouting the plasma. As we known, plasma has shielding effect on electromagnetic signals. An electromagnetic emitter 10 is adopted. The moment when it shields the electromagnetic signal when the plasma is ejected, the electromagnetic trigger 11 triggers the suppression timing trigger 6 at the same time.

This system mainly aims to control the time interval of electromagnetic wave emission and the time of controlling electromagnetic wave emission.

\subsection{Signal data collector}

The whole system is arranged in the same distance so that the variation of the time can reflect the sound speed's change, and then it shows the changes of stress and thrust. The hardware measurement of sound propagation time is mainly based on the timer to obtain the time interval between the transmitting moment and the receiving moment, so as to calculate the propagation speed of the ultrasonic wave. High precision implementation of time interval measurement is the core part of the signal data collector. When the transmitting probe 2 emits an ultrasonic wave, the chip of the data collector carries on a record to it, and when the receiving probe 7 receives the ultrasonic wave, the chip collects the primary signal again[19] [20] [21].

\subsection{Signal processing system}

The measurement result getting from the signal data collector is not the thrust but the time, so the important function of the signal processing system is to convert the time, the sound velocity, the stress and the thrust. This step can be realized through MATLAB.

The propagation time of each moment corresponds to one thrust. Meanwhile, the control system and the signal data collector ensure that the conversion thrust is approximate equal to the average thrust during the set time interval [22].

\section{Discussion}

In this paper, the acoustoelectric method, which has the advantages of simple structure, easy operation and high reliability, is first applied to the field of instantaneous thrust measurement field. Moreover, this method provides a basic idea for measuring the thrust of micro-thrusters of, can realize high precision and accurate measurement of instantaneous thrust and impulse. Micro thrust measuring methods of the non-contact measurement, and non-destructive measurement methods, does not require a sensor or other measuring device is directly installed on the structure, can reduce the interference of mechanical vibration and power supply cables for thrust measurement, solve the present commonly used micro thruster and micro impulse measuring device of zero drift, balance position stable, low accuracy and calibration difficult problem.

\section{References}


1. Haag T W 1991 Thrust stand for high - power electric propulsion devices Review of Scientific Instruments vol 62 chapter 5 pp 1186-91

2. Cubbin E, Ziemer J, Choueiri E and Jahn R 1997 Pulsed thrust measurements using laser interferometry Review of Scientific Instruments vol 68 chapter 6 pp 2339-46

3. Hallock A, Polzin K and Emsellem GD 2012 Thrust Stand Measurements of a Conical Pulsed Inductive Plasma Thruster

4. Zakrzwski C, Benson S, Sanneman P and Hoskins A 2002 On-Orbit Testing of the EO-1 Pulsed Plasma Thruster

5. Santoyo and Fernando Mendoza 1999 Simultaneous three-dimensional dynamic deformation measurements with pulsed digital holography Appl Opt vol 38 chapter 34 pp 7056-7062

6. Vladimir K, Aleksander L, Viktor M, Amitriy T and LevV S 2017 An estimation of the strainstress state under cyclic loading by the acoustoelasticity method Physics and Mathematics vol 3 pp 71-6

7. Astm S 2010 Standard Test Method for Verifying the Alignment of X-Ray Diffraction Instrument for Residual Stress Measurement

8. Soete $\mathrm{W}$ and R Vancrombrugge 1950 An industrial method for the determination of residual stresses Proc. SESA vol 8 chapter 1 pp 17-28

9. Eiras J, Vu Q, Lott M, Payá J, Garnier V and Payan C 2016 Dynamic acousto-elastic test using continuous probe wave and transient vibration to investigate material nonlinearity Ultrasonics vol 69 p 29

10. Si-Chaib M, Menad S, Djelouah $\mathrm{H}$ and Bocquet M 2001 An ultrasound method for the acoustoelastic evaluation of simple bending stresses $N d t$ and E International vol 34 chapter 8 pp $521-9$

11. Longhui Yin 2012 Ultrasonic stress measurement based on the LCR wave(in Chinese)

12. Knapp C and Carter G 2003 The generalized correlation method for estimation of time delay Transactions on Acoustics Speech \& Signal Processing vol 24 chapter 4 pp 320-7

13. Rose J and Nagy P 2004 Ultrasonic Waves in Solid Media Journal of the Acoustical Society of America vol 107 chapter 4 pp 1807-8

14. Cuihua Zhao 2013 Study on ultrasonic measurement of resudial stresses(in Chinese)

15. Haiquan Li, Lina Pan and Qingyan Liu 2000 Study and development of uitrasonic stress measurement system Journal of Test and Measurement Technology vol 22 chapter 7 pp 297299(in Chinese)

16. Nikitina $\mathrm{N}$ and Ostrovsky L 1998 An ultrasonic method for measuring stresses in engineering materials Ultrasonics vol 35 chapter 8 pp 605-10

17. Pao Y 1987 Theory of Acoustoelasticity and Acoustoplasticity

18. Okada K 1981 Acoustoelastic determination of stress in slightly orthotropic materials Experimental Mechanics vol 21 chapter 12 pp 461-6

19. Chaki $\mathrm{S}$ and $\mathrm{G}$ Bourse 2009 Stress level measurement in prestressed steel strands using acoustoelastic effect Experimental Mechanics vol 49 chapter 5 p 673

20. Wei Zhao 2002 On Acoustoelasticity of Surface Acoustic Wave for Ultrasonic NDT

21. Dike J, Johnson G and Flower E 1988 Acoustoelasticity Using Longitudinal Waves for Residual Stress Evaluation NDT and E International vol 28 p 118

22. Yaojie Huang 2015 Research on measurement method of welding residual stress in steel based on acoustoelasticity principle (in Chinese) 